\title{
Identification of Allosteric Nucleotide Sites of Tetramethylrhodamine-Labeled Aptamer for Noncompetitive Aptamer-Based Fluorescence Anisotropy Detection of a Small Molecule, Ochratoxin A
}

\author{
Qiang Zhao, ${ }^{\dagger}{ }^{\dagger}$ Qin $\mathrm{Lv}^{\dagger}$ and Hailin Wang ${ }^{\ddagger}$ \\ ${ }^{\dagger}$ Research Institute of Environmental Science, Shanxi University, Taiyuan, Shanxi Province, 030006, People’s Republic of China \\ ${ }^{\ddagger}$ State Key Laboratory of Environmental Chemistry and Ecotoxicology, Research Center for Eco-Environmental Sciences, Chinese \\ Academy of Sciences, Beijing, 100085, People's Republic of China
}

\section{Supporting Information}

\begin{abstract}
Aptamer-based fluorescence anisotropy (FA) assay combines the advantages of affinity aptamers in good stability, easy generation, and facile labeling and the benefits of FA in homogeneous analysis, such as robustness, simplicity, and high reproducibility. By using a fluorophore-labeled aptamer, FA detection of a small molecule is not as easy as detection of protein because the binding of a small molecule cannot cause significant increase of molecular weight of the dye-labeled aptamer. The intramolecular interaction between labeled tetramethylrhodamine (TMR) and DNA aptamer bases dramatically affects the local rotation and FA of TMR. This intramolecular interaction can be altered by aptamer conformation change upon target binding, leading to a significant change of FA of TMR. Taking this unique feature of a TMR-labeled aptamer, we described a noncompetitive aptamer-based fluorescence anisotropy assay for detection of small molecules by using ochratoxin A (OTA) as a model. We successfully identified the specific TMR-labeling sites of aptamers with sensitive FA response to OTA from the $5^{\prime}$-end, $3^{\prime}$-end and the internal thymine (T) bases. The aptamer with a TMR labeled on the 10th T base exhibited a remarkable FA reduction response to OTA $(\Delta r=0.078)$, without requiring any proteins or nanomaterials as FA signal enhancers. This FA approach for OTA showed high sensitivity with a detection limit of $3 \mathrm{nM}$, a dynamic range from $3 \mathrm{nM}$ to $3 \mu \mathrm{M}$, and good selectivity over the tested compounds with similar structures to OTA. The new strategy allowed the detection of OTA in diluted red wine and urine samples.
\end{abstract}

$\mathrm{F}$ luorescence anisotropy (FA) is a powerful and unique analytical technique for molecular interactions study and assay developments, which relies on the rotational diffusion of fluorescent tracers or probes under polarized excitation light. ${ }^{1-5}$ Fluorescence anisotropy shows strength in real-time analysis, simplicity, robustness, and sensitivity. FA assays have been applied to the studies about protein-protein interaction and protein-DNA interaction and the immunoassays for drug discovery, diagnostics, environmental monitoring, and food analysis. $^{1-7}$

The emergence of aptamers as affinity ligands makes the aptamer-based FA assay attractive due to their advantages over antibodies in ease of synthesis and labeling, great stability, good selectivity, and high binding affinity. ${ }^{8-12}$ Target-dependent allosteric structural change is one unique feature of an aptamer, and it allows the construction of an aptamer switch for target analysis through the adaptive transition converting a binding event into a detectable signal. ${ }^{8,13-17}$ The applications of fluorophore-labeled aptamers to direct FA analysis of proteins are relatively straightforward as aptamers are usually smaller than proteins in size. ${ }^{5,8,11,12}$ The binding of the fluorophorelabeled aptamers to proteins can cause enhanced FA signals by restricting the rotational diffusion of the labeled fluorophore as a result of increased molecular size. ${ }^{18-23}$

In contrast, the aptamer-based FA analysis of small molecules is not easy yet though a few efforts have been made. ${ }^{24-34}$ One approach requires a fluorescent tracer of the target to achieve competitive analysis of the small molecule, ${ }^{24}$ like the traditional fluorescence anisotropy immunoassay for small molecules, ${ }^{4}$ but it is difficult and time-consuming to conjugate a fluorophore to a small molecule without disrupting the affinity interaction between the small molecules and the affinity ligand. The direct aptamer-based FA approaches using dye-labeled aptamers for small molecules usually cannot give large FA responses because the binding of a small target only brings negligible changes in the molecular weight of the complex and the global rotation of the fluorescent label. One reported FA assay for small molecules relies on the binding-induced structure switch of the aptamer with an end-labeled dye (e.g., fluorescein or Texas red) and the subsequent change of the local rotation of the

Received: November 4, 2013

Accepted: December 19, 2013

Published: December 19, 2013 
Table 1. List of Tested DNA Oligonucleotides and Labeling Sites

\begin{tabular}{lc}
\hline Name & \multicolumn{1}{c}{ Sequences } \\
\hline O36 & 5'-GAT CGG GTG TGG GTG GCG TAA AGG GAG CAT CGG ACA-3' \\
5'-TMR-O36 ${ }^{\text {a }}$ & 5' (TMR)-GAT CGG GTG TGG GTG GCG TAA AGG GAG CAT CGG ACA-3' \\
T3-TMR-O36 & 5'-GAT(TMR) CGG GTG TGG GTG GCG TAA AGG GAG CAT CGG ACA-3' \\
T8-TMR-O36 & 5'-GAT CGG GT(TMR)G TGG GTG GCG TAA AGG GAG CAT CGG ACA-3' \\
T10-TMR-O36 & 5'-GAT CGG GTG T(TMR)GG GTG GCG TAA AGG GAG CAT CGG ACA-3' \\
T14-TMR-O36 & 5'-GAT CGG GTG TGG GT(TMR)G GCG TAA AGG GAG CAT CGG ACA-3' \\
T19-TMR-O36 & 5'-GAT CGG GTG TGG GTG GCG T(TMR)AA AGG GAG CAT CGG ACA-3' \\
T30-TMR-O36 & 5'-GAT CGG GTG TGG GTG GCG TAA AGG GAG CAT(TMR) CGG ACA-3' \\
3'-TMR-O36 & 5'-GAT CGG GTG TGG GTG GCG TAA AGG GAG CAT CGG ACA-3’'(TMR) \\
\hline
\end{tabular}

${ }^{a}$ The labeling sites are underlined and shown in red.

fluorescent dye, but only small FA increase is observed in most of cases. $^{25,26}$ Mass amplifying strategies recently have been exploited by using DNA oligos, ${ }^{27-29}$ DNA-binding proteins (e.g., single-stranded DNA binding (SSB) protein and thrombin), ${ }^{30-32}$ or nanomaterials ${ }^{33,34}$ to improve the fluorescence anisotropy response by increasing the change of the molecular size of the aptamer upon binding of small-molecule targets. In these approaches, competitive displacement or structure switching is usually involved. The use of proteins, complementary DNA oligos, or nanomaterials makes the assays complicated, and complex screening and rational designs are required.

It is known that distance-dependent intramolecular interaction between the fluorophore tetramethylrhodamine (TMR) and the surrounded bases (especially guanine bases) of DNA occurs in the TMR-labeled ssDNA and causes fluorescence quenching through a photoinduced electron-transfer (PET) mechanism. ${ }^{35-41}$ This intramolecular interaction restricts the local rotation of TMR on ssDNA and decreases the fluorescence lifetime of TMR, which affect the FA signal. ${ }^{20,22,38,42,43}$ It is also known that the binding of target usually induces an adaptive conformation change of the aptamer. $^{13-17}$ This conformational change can bring a subsequent alteration of the interaction between labeled TMR and the bases of the aptamer, and the FA of the labeled TMR on the aptamer can be significantly changed upon target binding. The unique intramolecular interaction between TMR and guanine bases and the allosteric property of the aptamer provide another opportunity to develop a simple FA strategy for target analysis with TMR-labeled aptamer, differing from the mass change dependent FA strategy. ${ }^{5,19,20,22,42,43}$ Considering that the binding of a small target cannot cause a large change of the molecular mass of the dye-labeled aptamer, this intramolecular interaction dependent FA strategy is more adaptable to small-molecule detection with a dye-labeled aptamer.
Here we describe a direct aptamer-based fluorescence anisotropy (FA) approach for small-molecule analysis with a TMR-labeled aptamer by using ochratoxin A (OTA) as a model. OTA is one of the fungal toxins found in a variety of foods, like grains, grapes, peanuts, and coffee, and can cause health risks to human being. ${ }^{44,45}$ The analysis of OTA is important in food safety, environmental analysis, and quality control. ${ }^{44,45}$ We introduced TMR on different sites of a 36-mer guanine $(\mathrm{G})$-rich DNA aptamer against OTA, ${ }^{46}$ including the internal thymine $(\mathrm{T})$ bases in the aptamer and the ends of the aptamer, to identify an FA-responsive labeling site for great FA change upon OTA binding. Among the TMR-labeled aptamers, the aptamer with TMR labeled on the 8th, 10th, or 19th T base could show large remarkable decrease of FA of TMR in the presence of OTA due to the change of the intramolecular interaction between the labeled TMR and the aptamer bases. The best FA response to OTA was obtained by using the aptamer with a TMR labeled on the 10th T base. A simple and sensitive FA detection of OTA was achieved with this favorable TMR-labeled aptamer probe. The experimental conditions that affect the performance of the FA assay for OTA (e.g., cations, $\mathrm{pH}$, and temperature) were optimized in detail. This work shows that a simple and direct aptamer-based FA assay for small molecules can be achieved with a TMR-labeled aptamer by taking advantage of the intramolecular interaction between TMR and aptamer bases and the unique feature of aptamer in target-induced fit binding.

\section{EXPERIMENTAL SECTION}

Materials and Reagents. OTA, $N$-acetyl-L-phenylalanine (NAP), 7-amino-4-methyl coumarin (AMC), and warfarin were ordered from Sigma. Phenylalanine, arginine, aspartic acid, serine, and tyrosine were obtained from Amresco. All the DNA oligonucleotides were custom-synthesized, labeled, and purified by Sangon Biotech (Shanghai, China). The DNA aptamer against OTA had the following sequence: $5^{\prime}$-GAT CGG GTG TGG GTG GCG TAA AGG GAG CAT CGG ACA-3' 
(denoted as $\mathrm{O} 36$ in this work). ${ }^{46}$ DNA aptamers with a single TMR at different labeling sites are listed in Table 1. The DNA as control, denoted as 5'-TMR-S36, had the following sequence: 5'-CAT CCC CTC TCG CTC CCC TAG ACC CAC CAT CCC GCA-3', and TMR was labeled on $5^{\prime}$-end. The aptamers with other fluorophore labels on the 10th internal thymine of the aptamer, like fluorescein (FAM) or Texas red (TXR), were also synthesized, which were denoted as T10-FAM-O36 and T10-TXR-O36, respectively (Table 1). Red wine was obtained from a local supermarket. Ultrapure water for solution preparation was obtained through a Purelab Ultra Elga Labwater system. All other reagents were of analytical grade.

Fluorescence Measurement. Fluorescence anisotropy (FA) analysis was conducted on JASCO FP-8300 fluorescence spectrometer (Japan) equipped with a thermostat for precise temperature control. The fluorescence anisotropy $(r)$ is defined as $r=\left(I_{\mathrm{VV}}-G I_{\mathrm{VH}}\right) /\left(I_{\mathrm{VV}}+2 G I_{\mathrm{VH}}\right)$, where $I$ corresponds to the emission intensity, and the subscripts $\mathrm{V}$ and $\mathrm{H}$ mean the vertical and horizontal orientation of the polarizer, respectively. The orientations of the excitation and the emission polarizers are shown by the first and the second subscripts, respectively. The fluorescence anisotropy is given as $r=\left(I_{\mathrm{VV}}-G I_{\mathrm{VH}}\right) /\left(I_{\mathrm{VV}}+\right.$ $\left.2 G I_{\mathrm{VH}}\right)$. The instrumental correction factor $\left(G=I_{\mathrm{HV}} / I_{\mathrm{HH}}\right)$ was used to correct the wavelength response to polarization of the emission optics and detectors. For experiments measuring the FA of TMR-labeled DNA oligos, FA was tested with an excitation at $560 \mathrm{~nm}$ and an emission at $578 \mathrm{~nm}$. When FA of other fluorophores labeled on aptamers was tested, the measurements were conducted with the excitation at $498 \mathrm{~nm}$ (FAM label) or $588 \mathrm{~nm}$ (TXR label) and the emission at 518 $\mathrm{nm}$ (FAM label) or $608 \mathrm{~nm}$ (TXR label). Slits for the excitation and the emission were both set at $5 \mathrm{~nm}$. A $100 \mu \mathrm{L}$ quartz cuvette was employed as the sample cell. Without further statement, the FA measurement experiments were all performed at $20{ }^{\circ} \mathrm{C}$. For each FA measurement, five data points were collected, and the average value was used for data processing.

The FA assay for OTA by using the dye-labeled aptamer was performed by the following procedure. Dye-labeled aptamer at $27 \mathrm{nM}$ was mixed with varying concentrations of OTA in the binding buffer solution containing $10 \mathrm{mM}$ Tris- $\mathrm{HCl}(\mathrm{pH} 8.5)$, $120 \mathrm{mM} \mathrm{NaCl}, 20 \mathrm{mM} \mathrm{CaCl}$, and $0.1 \%$ Tween 20 in a final volume of $200 \mu \mathrm{L}$, and the FA of the dye labeled on aptamers was recorded.

Fluorescence intensity measurement was finished on a JASCO FP-8300 fluorescence spectrometer (Japan). The fluorescence intensity of TMR with an excitation at $560 \mathrm{~nm}$ was recorded from 572 to $650 \mathrm{~nm}$.

\section{RESULTS AND DISCUSSION}

Identification of Allosteric and FA-Responsive Nucleotide Sites of Anti-OTA Aptamer upon OTA Binding. It has been known that labeled TMR can intramolecularly interact with the adjacent $G$ bases of the ssDNA and cause an effective fluorescence quenching through a photoinduced electron-transfer mechanism. ${ }^{35-41}$ Among four bases of DNA (A, T, G, and C), only guanine bases can act as an effective electron donor to a fluorophore acceptor. ${ }^{37,38} \mathrm{G}$ bases are involved in many aptamer sequences, ${ }^{8-12}$ and it is also found that target binding usually induces a structure change of the aptamer. ${ }^{13-17}$ Therefore, when TMR is introduced on a specific site of an aptamer, a structure-dependent molecular interaction between TMR and G bases of the aptamer may occur. Then, the target binding may induce a change of the TMR-involved intramolecular interaction followed by the alteration of the rotational diffusion and fluorescence lifetime of TMR, and thus a remarkable FA change can be triggered upon target binding. ${ }^{2,5,43}$ Taking these unique properties of TMR and aptamer, a simple direct FA strategy for small-molecule detection can be established. We chose ochratoxin A (OTA) as a model of a small molecule to propose this simple FA strategy for small-molecule analysis by using a TMR-labeled aptamer.

A TMR dye is conjugated on a G-rich 36-mer DNA aptamer against OTA, and TMR may interact with the spatially surrounded $G$ bases of the aptamer depending on the labeling sites. The binding of OTA to the aptamer brings a change of aptamer conformation. It is expected that when OTA binding induces diminishment of the interaction between TMR and the $\mathrm{G}$ bases of the aptamer, enhancing the freedom and fluorescence lifetime of TMR, a decreased FA of TMR will be observed (Scheme 1), while an increased FA of TMR can be

Scheme 1. Schematic Diagram for Target-Binding Induced Changes of Intramolecular Interaction of TMR and Fluorescence Anisotropy of TMR-Labeled Aptamer ${ }^{a}$
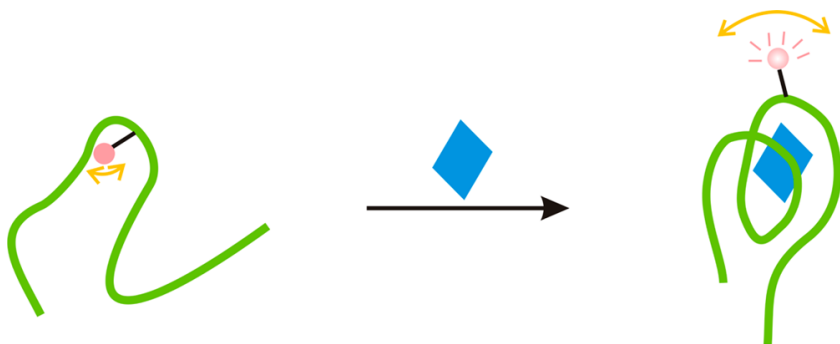

${ }^{a}$ TMR is labeled on a specific site of the aptamer. The binding of a small target induces the conformation change of the TMR-labeled aptamer followed by the alteration of the intramolecular interaction between TMR and aptamer and the subsequent local rotation of TMR, resulting in a fluorescence anisotropy change of the TMR-labeled aptamer.

produced when OTA binding tightens the intramolecular interaction between TMR and the G bases. If the status of TMR in intramolecular interactions is not changed by target binding, negligible FA responses will be observed. The screened FA-responsive aptamer probe can be used to detect OTA by measuring the FA changes induced by OTA.

We tested the FA responses of the TMR-labeled aptamers to OTA to identify the FA-responsive sites of the aptamer upon OTA binding. The labeling sites included the $5^{\prime}$-end, $3^{\prime}$-end, and internal thymine bases (T3, T8, T10, T14, T19, and T30) of a 36-mer aptamer against OTA, shown in Table 1. In the absence of OTA, most of these TMR-labeled aptamers exhibited a high FA value of more than 0.210 (Figure 1A). In the absence of OTA, the FA value of the control DNA (5'TMR-S36) and $3^{\prime}$-TMR-O36 was about 0.146 and 0.178 , respectively. Upon addition of OTA, these TMR-labeled aptamers displayed distinct FA responses (shown in Figure S1 in the Supporting Information and Figure 1B). The 5'TMR-S36 control did not show a remarkable change in FA. The T10-TMR-O36 exhibited great FA reduction to OTA, and the FA was decreased from $\sim 0.243$ to $\sim 0.165$, with a maximum FA reduction about 0.078. The T8-TMR-O36, T19-TMR-O36, 

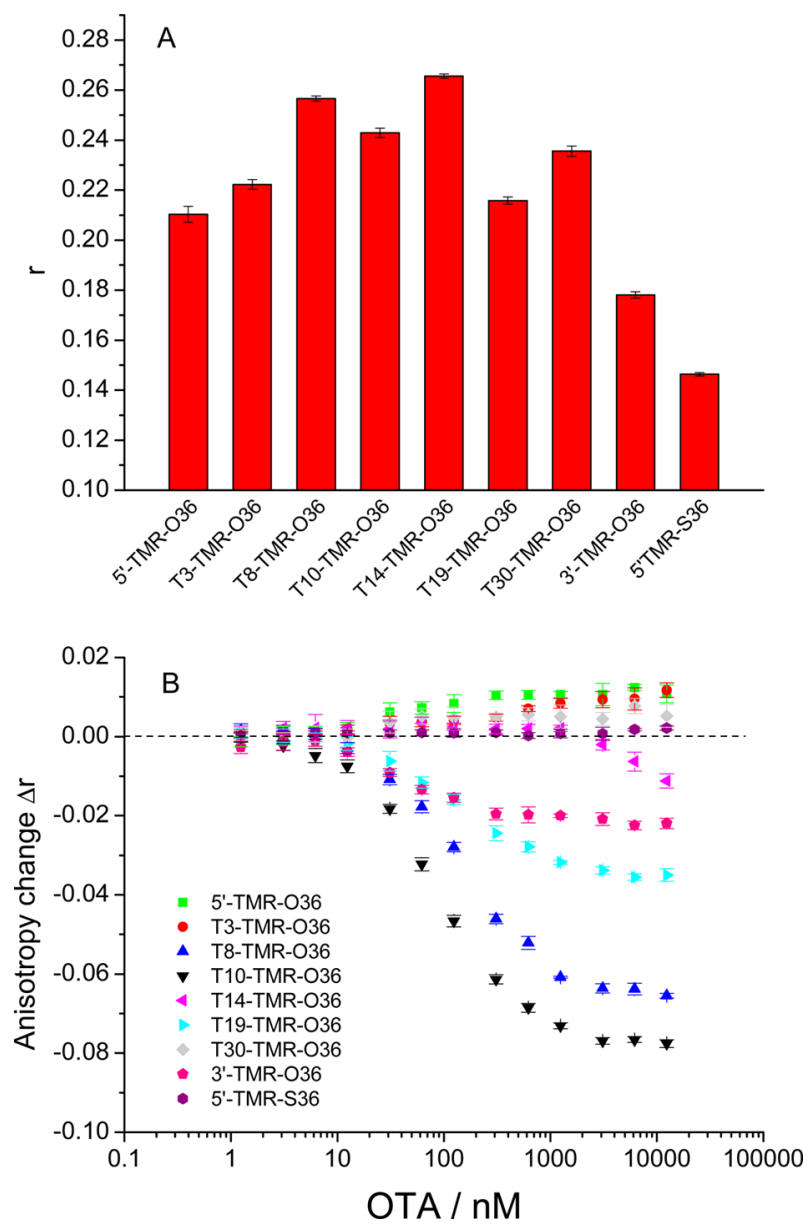

Figure 1. (A) Fluorescence anisotropy values of TMR-labeled aptamers in the absence of OTA. (B) Fluorescence anisotropy changes of TMR-labeled aptamers in response to varying concentrations of OTA.

and 3 '-TMR-O36 also showed FA decreases in response to OTA (Figure 1B), and the maximum FA decreases for T8TMR-O36, T19-TMR-O36, and 3'-TMR-O36 were about $0.065,0.035$, and 0.022 , respectively. The T14-TMR-O36 did not show significant FA responses to OTA at concentrations lower than $1 \mu \mathrm{M}$. The TMR-labeled aptamer probes of $5^{\prime}$ TMR-O36, T3-TMR-O36, and T30-TMR-O36 only showed slight FA increase in the presence of OTA (Figure 1B). The observed significant FA changes of the TMR-labeled aptamers cannot be attributed to the change in molecular size of complex because the molecular weight of OTA (MW $403 \mathrm{Da})$ is negligible compared to that of the TMR-labeled aptamers (MW $\sim 11-12 \mathrm{kDa})$.

We measured the fluorescence intensity of TMR-labeled aptamers upon the addition of OTA to assess whether the FA change was related with the alteration of the fluorescence intensity of TMR (shown in Figure S2 in the Supporting Information). The fluorescence intensity of T10-TMR-O36 increased with addition of OTA, and the maximum increase in fluorescence intensity was about $106 \%$ over the blank value. T8-TMR-O36 and T19-TMR-O36 also showed increased fluorescence intensity in the presence of OTA with the maximum increases about $122 \%$ and $57 \%$, respectively. T3TMR-O36, T30-TMR-O36, and 5'-TMR-O36 exhibited slightly decreased fluorescence intensity of TMR upon OTA addition, and the maximum decrease in fluorescence intensity was only about $36 \%$ for T3-TMR-O36 or $15 \%$ for $5^{\prime}-$ TMR-O36 and T30-TMR-O36. 3'-TMR-O36, T14-TMR-O36, and 5'TMR-S36 did not show significant change of fluorescence intensity with increasing concentrations of OTA.

For T8-TMR-O36, T10-TMR-O36, and T19-TMR-O36, the OTA-binding induced fluorescence anisotropy change is inversely proportional to the increase of fluorescence intensity of these TMR-labeled aptamers (Figure S3 in the Supporting Information). The dramatic FA decrease accompanied significant increase of fluorescence intensity indicates that the target-binding induced change of the intramolecular interaction leads to the alteration of FA of these TMR-labeled aptamers. The low fluorescence intensity of TMR-labeled aptamer suggests that fluorescence quenching of TMR occurs due to the intramolecular interactions between TMR and the adjacent $\mathrm{G}$ bases of the aptamer prior to OTA binding, ${ }^{37}$ causing a low local rotation of TMR and a high FA. In contrast, the high fluorescence intensity of TMR-labeled aptamers means that the intramolecular interaction between TMR and G bases of the aptamer upon OTA binding is eliminated, ${ }^{37}$ resulting in a large local rotation of TMR and a low FA.

Effect of TMR Labeling on Binding Affinity of AntiOTA Aptamer. To further test whether the insignificant FA changes of some TMR-labeled aptamers in response to OTA are due to the binding affinity decrease or the insignificant changes of intramolecular interaction between TMR and aptamer, we analyzed the binding affinity of the TMR-labeled aptamer by using a previously reported FA approach (shown in the Supporting Information). ${ }^{47,48}$ This approach relies on the measurement of FA of OTA at a fixed concentration in the presence of increasing concentrations of aptamer, taking advantage of the intrinsic fluorescence of OTA (Figures S4 and S5 and Table S1 in the Supporting Information). ${ }^{47}$

In comparison to the unlabeled aptamer (O36) with good binding affinity to OTA $\left(K_{\mathrm{d}} \sim 23 \mathrm{nM}\right)$, the $5^{\prime}$-TMR-S36 control and T14-TMR-O36 showed little binding affinity to OTA, so the FA responses of these two DNA probes to OTA were negligible. T3-TMR-O36 exhibited greatly reduced binding affinity $\left(K_{\mathrm{d}} \sim 555 \mathrm{nM}\right)$, and its FA response to OTA was not large. 5'-TMR-O36 and T30-TMR-O36 both maintained good binding affinity to OTA, but they did not show remarkable FA responses to OTA, meaning both of them lacked large changes of the TMR-involved intramolecular interaction and the local rotation of TMR upon OTA binding. Among the TMR-labeled aptamers showing FA reduction responses to OTA, T10-TMR-O36, T8-TMR-O36, and T19TMR-O36 still showed comparable binding affinity to OTA, with dissociation constants ranging from 153 to $262 \mathrm{nM}$, while $3^{\prime}$-TMR-O36 showed a better binding affinity $\left(K_{\mathrm{d}} \sim 58 \mathrm{nM}\right)$ than the other three TMR-labeled aptamers, but only a small OTA-induced FA decrease was observed for 3'-TMR-O36. Considering the low FA value of $3^{\prime}$-TMR-O36 in the absence of OTA and negligible change in fluorescence intensity upon the addition of OTA, it suggests that the intramolecular interaction between TMR and the bases in the $3^{\prime}-\mathrm{TMR}-\mathrm{O} 36$ is weak. It is likely that the FA change of $3^{\prime}$-TMR-O36 in the presence of OTA is not caused by the change of intramolecular interaction between OTA and G bases. Figure 2 summarizes the FA values of TMR-labeled aptamers in the absence of OTA, FA changes of the TMR-labeled aptamers induced by OTA at $12 \mu \mathrm{M}, K_{\mathrm{d}}$ 's of the TMR-labeled aptamers, and the changes in $K_{\mathrm{d}}$ caused by TMR labeling over the $K_{\mathrm{d}}$ of the unlabeled 


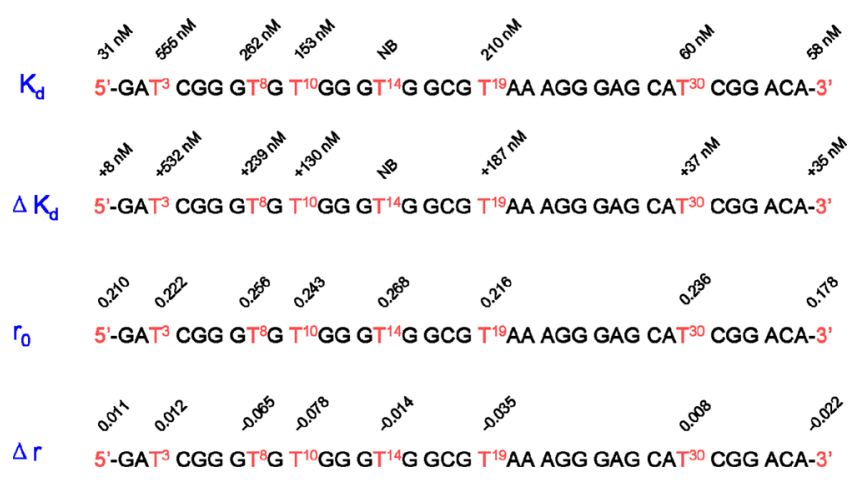

Figure 2. Comparison of the binding affinity and FA responses of TMR-labeled aptamers to OTA with respect to the labeling sites (shown in red). Dissociation constant of the TMR-labeled aptamers $\left(K_{\mathrm{d}}\right)$, the TMR-labeling caused $K_{\mathrm{d}}$ change over the $K_{\mathrm{d}}$ of the unlabeled aptamer $\left(\Delta K_{\mathrm{d}}\right)$, the FA value in the absence of OTA $\left(r_{0}\right)$, and the obtained maximum FA change caused by OTA $(\Delta r)$ were shown near the labeling sites of the aptamer sequence. NB meant no binding affinity.

aptamer, with respect to the different TMR labeling sites of the aptamer.

Effect of Sorts of Labeled Fluorophores on FA Response. The nature of the labeled fluorophores also has effects on the FA response of the dye-labeled aptamer to OTA due to the different intrinsic properties of the dyes. Aptamer with FAM or TXR labeled on 10th T in the anti-OTA aptamer O36 (T10-FAM-O36 or T10-TXR-O36) was tested (Figure S6 in the Supporting Information). The T10-FAM-O36 did not show a large FA response to OTA, though its fluorescence intensity increased with the addition of OTA. The maximum fluorescence increase of T10-FAM-O36 was about $33 \%{ }^{48}$ In the absence of OTA, the FA value of T10-FAM-O36 was around 0.090, much lower than the FA value of T10-TMR-O36 $(\sim 0.243)$. This observation is in agreement with the previous work which reports that the labeled FAM shows a high level of local rotation and causes low FA value. ${ }^{20,26,38,39}$ The high local mobility of FAM can be attributed to the weak interaction between FAM and the bases of the aptamer as the FAM has negative charges and is electrostatically repelled from the negatively charged DNA. ${ }^{38,39}$ The higher local mobility of the labeled FAM than the labeled TMR possibly makes the T10FAM-O36 show less sensitive FA response to OTA. ${ }^{20,38,39}$ The longer fluorescence lifetime of FAM than $\mathrm{TMR}^{43}$ and the less quenching extent of FAM by aptamer bases may also cause the insignificant FA change of T10-FAM-O36 in response to OTA. $^{38,39,43}$

The T10-TXR-O36 showed small FA reduction response to OTA. The FA of T10-TXR-O36 was around 0.198 in the absence of OTA. For T10-TXR-O36, the maximum FA decrease caused by OTA was only about 0.020 , lower than that obtained by T10-TMR-O36. In the presence of OTA, the fluorescence intensity of T10-TXR-O36 showed slight decrease at high concentrations of OTA, with less than $10 \%$ maximum decrease (Figure S6 in the Supporting Information). TXR is a cationic dye with a similar structure to TMR, and can interact with the DNA, and the labeled TXR is coupled with the rotational mobility of the aptamer, so a high FA value of T10TXR-O36 in the absence of OTA is observed, differing from the labeled FAM. ${ }^{20,26,32,38,39}$ However, TXR does not undergo the photoinduced electron transfer to $G$ bases due to the low reduction potential for TXR, and little fluorescence quenching occurs during interactions, which is different from TMR. ${ }^{38,39}$ Considering the negligible change of fluorescence intensity and small FA reduction response of TXR upon OTA, it is likely that the FA decrease of T10-TXR-O36 upon OTA is caused by the conformation change of the aptamer instead of the change of intramolecular interaction between dye and aptamer bases, similar to $3^{\prime}$-TMR-O36.

In addition, T10-FAM-O36 and T10-TXR-O36 both showed good binding affinity to OTA (Table S1 in the Supporting Information), excluding the possibility that the binding affinity decrease leads to the less sensitive FA response to OTA. These results show the fluorophores labeled on the same site of the aptamer display distinct behaviors in response to OTA binding, significantly depending on the photophysical properties and molecular structures of the fluorophores. ${ }^{20,26,32,38,39,43}$ Clearly, TMR-labeled aptamer is preferred for OTA analysis as it can give more sensitive FA response to OTA. On the basis of the above investigation, T10-TMR-O36 was chosen as a favorable aptamer probe in FA analysis of OTA.

Optimization of FA Analysis for OTA. We investigated the influence of experimental conditions on FA responses of T10-TMR-O36 to OTA, such as cations, buffer $\mathrm{pH}$, temperature, and incubation time. First, we tested the effect of $\mathrm{CaCl}_{2}$ on the FA responses of T10-TMR-O36 in the solution containing $10 \mathrm{mM}$ Tris- $\mathrm{HCl}(\mathrm{pH} 8.5)$ and $120 \mathrm{mM} \mathrm{NaCl}$ (see Figure $\mathrm{S} 7$ in the Supporting Information). $\mathrm{CaCl}_{2}$ did not cause large change of FA of T10-TMR-O36 in the absence of OTA. In the presence of OTA the absolute FA change of T10TMR-O36 caused by OTA gradually increased with the addition of $\mathrm{CaCl}_{2}$. When $\mathrm{CaCl}_{2}$ concentration was higher than $40 \mathrm{mM}$ in the binding buffer solution, the FA change became smaller. These results can be explained by the fact that $\mathrm{CaCl}_{2}$ at optimal concentrations is needed for good binding affinity of the aptamer to OTA, reported by the early study. ${ }^{47,48}$ The addition of $20 \mathrm{mM} \mathrm{CaCl}_{2}$ in the binding buffer solution was applied to the further experiments.

The concentration of $\mathrm{NaCl}$ in the binding buffer solution also exhibited influence on FA response of T10-TMR-O36 to OTA (see Figure S8 in the Supporting Information). The FA change induced by OTA over the blank was increased with the addition of $\mathrm{NaCl} . \mathrm{NaCl}$ at $120 \mathrm{mM}$ in the solution is favorable for large FA change, as the simultaneous presence of $\mathrm{NaCl}$ and $\mathrm{CaCl}_{2}$ can enhance the binding strength of the anti-OTA aptamer. $^{47}$

When the $\mathrm{pH}$ of the binding buffer solution was lower than 5.0, a weak absolute FA change caused by OTA was observed (shown in Figure S9 in the Supporting Information) as low $\mathrm{pH}$ decreased the binding affinity of the aptamer. ${ }^{46}$ Large FA changes were obtained in the binding buffer solution with $\mathrm{pH}$ ranging from 5.0 to 9.5 . Binding buffer solution at $\mathrm{pH} 8.5$ was chosen in our FA analysis as the maximum OTA-induced FA change was achieved.

To evaluate the influence of temperature, FA analysis of OTA with T10-TMR-O36 was conducted at temperature ranging from 4 to $60{ }^{\circ} \mathrm{C}$ (Figure 3). The blank FA value from the aptamer probe in the absence of OTA decreased with the temperature increase as a result of increase of the local motion of the labeled TMR. At temperatures below $30{ }^{\circ} \mathrm{C}$, the aptamer probe showed almost the same FA decrease upon OTA (Figure S10 and Table S2 in the Supporting Information). When the temperature was higher than $30{ }^{\circ} \mathrm{C}$ the absolute FA change of T10-TMR-O36 caused by OTA decreased sharply with the increase of temperature. FA response of T10-TMR-O36 to 

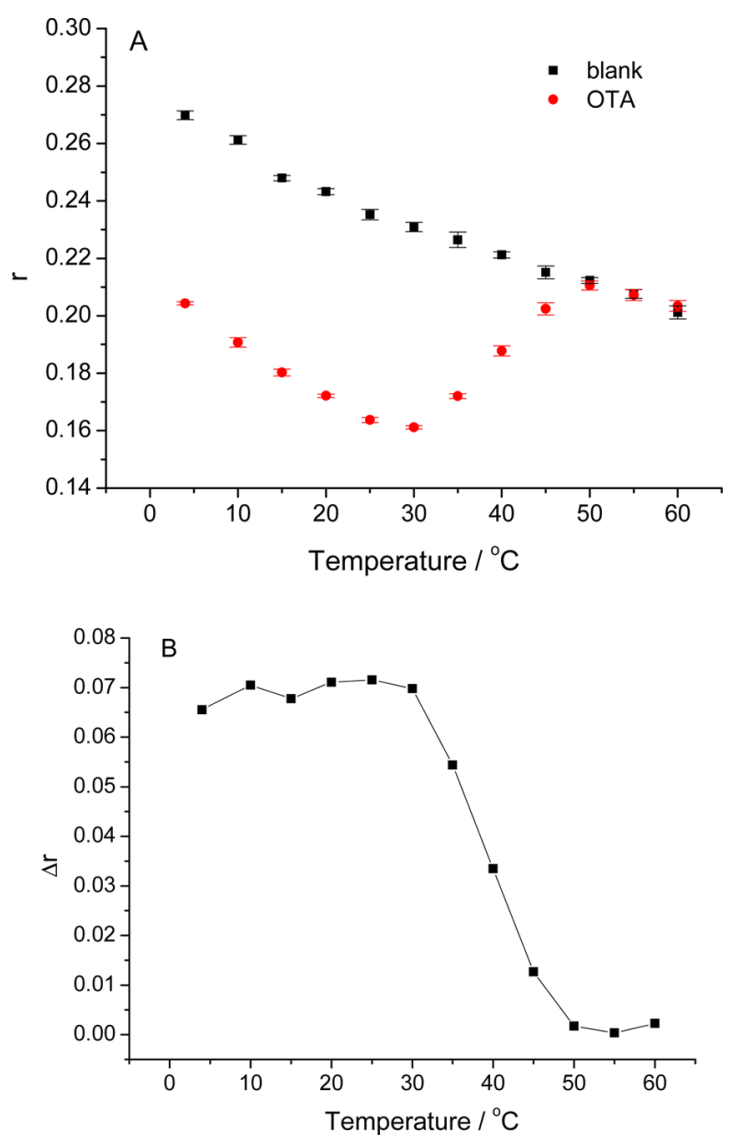

Figure 3. (A) Effects of temperature on the FA responses of T10TMR-O36 in the absence or the presence of OTA $(620 \mathrm{nM})$ in the binding buffer solution containing $10 \mathrm{mM}$ Tris- $\mathrm{HCl}(\mathrm{pH} \mathrm{8.5),} 120$ $\mathrm{mM} \mathrm{NaCl}, 20 \mathrm{mM} \mathrm{CaCl}_{2}$, and $0.1 \%$ Tween 20. (B) The absolute FA change caused by OTA $(620 \mathrm{nM})$ vs the applied temperature.

OTA completely disappeared at $50{ }^{\circ} \mathrm{C}$, which suggested that a temperature higher than $30{ }^{\circ} \mathrm{C}$ could not be applied to the FA assay for OTA. The high temperature greatly reduced the binding strength of the aptamer to OTA (Table S2 in the Supporting Information). Thus, FA analysis of OTA at $20{ }^{\circ} \mathrm{C}$ was performed for good sensitivity and easy handling.

The effect of incubation time on FA responses of the aptamer probe T10-TMR-O36 to OTA was also studied. A fast FA decrease was observed after a $1 \mathrm{~min}$ incubation of T10TMR-O36 and OTA. More than 40 min of incubation at room temperature could produce a larger FA decrease, meaning the binding process needed enough incubation time to get a complete equilibrium (Figure $\mathrm{S} 11$ in the Supporting Information). To get a better sensitivity, a 40 min incubation of sample mixture was applied prior to FA analysis.

Under the optimized condition, the FA assay showed a detection limit of OTA around $3 \mathrm{nM}$ based on a signal-to-noise ratio $>3$, corresponding to about $1 \mathrm{ng} / \mathrm{mL}$ (Figure 4). The dynamic detection range was from 3 to $3100 \mathrm{nM}$, covering about 3 orders of magnitude. The relative standard deviation (RSD) for five repeated analyses was less than 3\%. The performance of the other TMR-labeled aptamers is listed in Table S3 in the Supporting Information. Detection of OTA could also be achieved by measuring the change of fluorescence intensity of T10-TMR-O36 with a detection limit about $6 \mathrm{nM}$ (Figure S2 in the Supporting Information). However, the fluctuation of fluorescence intensity resulted from concen-

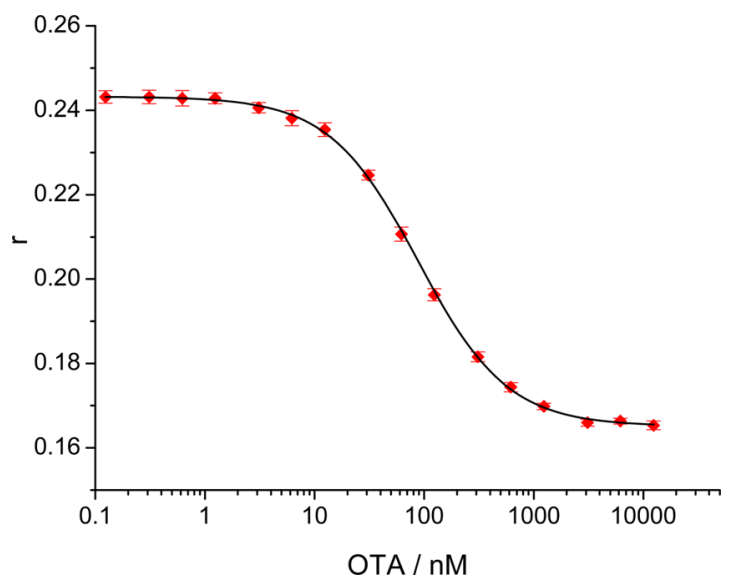

Figure 4. Fluorescence anisotropy of T10-TMR-O36 in response to varying concentrations of OTA.

tration variance, photobleaching, and instrument changes in the direct fluorescence intensity measurement may cause limitations in reproducibility. In contrast, as a ratiometric technique, FA measurement is insensitive to photobleaching of fluorophores and fluorescence intensity change caused by the source fluctuation, showing advantages over the direct fluorescence intensity measurement in reproducibility and precision.

Assay Selectivity and Performance in Complex Sample Matrix. We examined the selectivity of the T10TMR-O36-based direct FA assay for OTA by detection of OTA analogues including $\mathrm{N}$-acetyl-L-phenylalanine (NAP), 7-amino4-methyl coumarin (AMC), and a few amino acids including arginine, phenylalanine, aspartic acid, serine, and tyrosine (Figure S12 in the Supporting Information), with the T10TMR-O36. No significant FA changes were observed when these tested compounds existed in solutions at very high concentrations (Figure 5). The simultaneous presence of the tested compounds at high concentrations and OTA did not cause interference. This result shows the FA analysis of OTA is highly specific.

To test the feasibility of the FA assay for the OTA in solution containing organic solvent, we also analyzed the OTA spiked in diluted methanol, ethanol, or dimethyl sulfoxide (DMSO), because the detection of OTA in real samples usually needs the preconcentration of OTA and the subsequent elution of the OTA with methanol or other organic solvent prior to analysis. ${ }^{44-46,49}$ The presence of $20 \%$ methanol, 20\% ethanol, or $20 \%$ DMSO did not exhibit great effects on OTA detection (Figure S13A in the Supporting Information).

We further challenged the FA assay for OTA by testing the performance of the assay for OTA spiked in more complicated sample matrixes, like red wine and human urine samples. When OTA was spiked in the 50-fold-diluted red wine, the T10TMR-O36 still showed sensitive response to OTA (Figure S13B in the Supporting Information). The detection limit of OTA spiked in 50-fold-diluted red wine was about $12 \mathrm{nM}$. In the 10-fold-diluted human urine samples, the spiked OTA was detected with comparable sensitivity to that in the binding buffer solution (Figure S13B in the Supporting Information). The results show that this FA approach is feasible for OTA analysis in a complicated sample matrix.

When compared with other methods for OTA detection using aptamers, ${ }^{28,45,50-56}$ our strategy shows advantages such as 


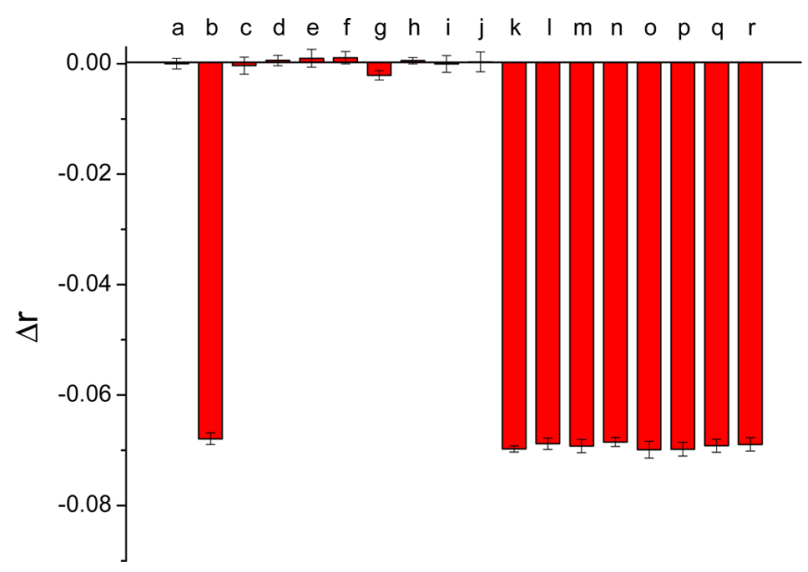

Figure 5. Selectivity of the aptamer probe T10-TMR-O36 for OTA analysis. FA responses of T10-TMR-O36 were tested in the absence of OTA (a) or in the presence of $620 \mathrm{nM}$ OTA (b), $10 \mu \mathrm{M}$ NAP (c), 7 $\mu \mathrm{M}$ warfarin (d), $9 \mu \mathrm{M}$ AMC (e), $62 \mu \mathrm{M}$ arginine (f), $62 \mu \mathrm{M}$ phenylalanine (g), $62 \mu \mathrm{M}$ aspartic acid (h), $62 \mu \mathrm{M}$ serine (i), or 62 $\mu \mathrm{M}$ tyrosine $(\mathrm{j})$. The FA responses of T10-TMR-O36 were also tested in the simultaneous presence of $620 \mathrm{nM}$ OTA and the tested compounds like $10 \mu \mathrm{M}$ NAP (k), $7 \mu \mathrm{M}$ warfarin (l), $9 \mu \mathrm{M}$ AMC (m), $62 \mu \mathrm{M}$ arginine (n), $62 \mu \mathrm{M}$ phenylalanine (o), $62 \mu \mathrm{M}$ aspartic acid (p), $62 \mu \mathrm{M}$ serine (q), or $62 \mu \mathrm{M}$ tyrosine (r).

high reproducibility, simplicity, sensitivity, and potential of high-throughput analysis. In our method only one dye-labeled aptamer is needed, much simpler than the competitive FA assays requiring the fluorescent tracers of OTA, ${ }^{57,58}$ fluorescent complementary oligos, ${ }^{28}$ or other reagents for signal amplification (e.g., proteins or nanomaterials). ${ }^{31-34}$ The comparison in assay performance with other FA assays for OTA and aptamer-based FA assays for small molecules is summarized in Table S4 in the Supporting Information. $^{25-29,31-34,57,58}$

\section{CONCLUSION}

In summary, we demonstrated a simple fluorescence anisotropy strategy for direct analysis of a small molecule, OTA, with a TMR-labeled anti-OTA aptamer. It relied on the changes of the intramolecular interaction between TMR and guanine bases of aptamer and the subsequent local rotation of TMR upon target binding. By investigating the labeling sites including the 3 '-end, 5 -end, and the internal thymine bases of the aptamer, we successfully identified the FA-responsive labeling sites to OTA binding. One aptamer with TMR labeled on the 10th thymine base showing large FA decrease response upon OTA was used to develop a sensitive FA assay for OTA, exhibiting good selectivity and a wide dynamic range, and OTA at $3 \mathrm{nM}$ could be detected. This assay takes advantage of FA in simplicity and robustness and also combines the unique merits of an affinity aptamer in good stability, ease of production, ease of labeling, and the binding-induced allosteric property. Considering the prevalence of guanine residues in many aptamers and targetbinding induced conformational change of aptamers, it is expected that this FA strategy using TMR-labeled aptamers can be applied to other small molecules.

\section{ASSOCIATED CONTENT}

\section{S Supporting Information}

Additional information as noted in text. This material is available free of charge via the Internet at http://pubs.acs.org.

\section{AUTHOR INFORMATION}

\section{Corresponding Author}

*Phone: 86-351-7011011. Fax 86-351-7011011. E-mail: chemzhaoq@hotmail.com.

Notes

The authors declare no competing financial interest.

\section{ACKNOWLEDGMENTS}

This work was supported by the grants from National Natural Science Foundation of China (Grant Nos. 21222503, 21105125), the State Key Laboratory of Environmental Chemistry and Ecotoxicology in the Research Center for Eco-Environmental Sciences of the Chinese Academy of Sciences (Grant No. KF2010-24), and the Key Project of Chinese Ministry of Education (Grant No. 212020). This work was also supported by Outstanding Youth Talents Program of Shanxi Province.

\section{REFERENCES}

(1) Gradinaru, C. C.; Marushchak, D. O.; Samim, M.; Krull, U. J. Analyst 2010, 135, 452-459.

(2) Jameson, D. M.; Ross, J. A. Chem. Rev. 2010, 110, 2685-2708.

(3) Lea, W. A.; Simeonov, A. Expert Opin. Drug Discovery 2011, 6, $17-32$.

(4) Smith, D. S.; Eremin, S. A. Anal. Bioanal. Chem. 2008, 391, 1499-1507.

(5) Lakowicz, J. R. Principles of Fluorescence Spectroscopy, 3rd ed.; Springer: New York, 2006.

(6) Yang, P.; Whelan, R. J.; Jameson, E. E.; Kurzer, J. H.; Argetsinger, L. S.; Carter-Su, C.; Kabir, A.; Malik, A.; Kennedy, R. T. Anal. Chem. 2005, 77, 2482-2489.

(7) Wan, Q. H.; Le, X. C. Anal. Chem. 2000, 72, 5583-5589.

(8) Liu, J.; Cao, Z.; Lu, Y. Chem. Rev. 2009, 109, 1948-1998.

(9) Klussmann, S., Ed. The Aptamer Handbook, Functional Oligonucleotides and Their Applications; Wiley-VCH: Weinheim, Germany, 2006.

(10) Li, Y., Lu, Y., Eds. Functional Nucleic Acids for Analytical Applications; Springer: New York, 2009.

(11) Jayasena, S. D. Clin. Chem. 1999, 45, 1628-1650.

(12) McGown, L. B.; Joseph, M. J.; Pitner, J. B.; Vonk, G. P.; Linn, C. P. Anal. Chem. 1995, 67, 663A-668A.

(13) Hermann, T.; Patel, D. J. Science 2000, 287, 820-825.

(14) Lubin, A. A.; Plaxco, K. W. Acc. Chem. Res. 2010, 43, 496-505.

(15) Jhaveri, S. D.; Kirby, R.; Conrad, R.; Maglott, E. J.; Bowser, M.; Kennedy, R. T.; Glick, G.; Ellington, A. D. J. Am. Chem. Soc. 2000, 122, 2469-2473.

(16) Nutiu, R.; Li, Y. Chem.-Eur. J. 2004, 10, 1868-1876.

(17) Juskowiak, B. Anal. Bioanal. Chem. 2011, 399, 3157-3176.

(18) Potyrailo, R. A.; Conrad, R. C.; Ellington, A. D.; Hieftje, G. M. Anal. Chem. 1998, 70, 3419-3425.

(19) Fang, X.; Cao, Z.; Beck, T.; Tan, W. Anal. Chem. 2001, 73, $5252-5757$.

(20) Gokulrangan, G.; Unruh, J. R.; Holub, D. F.; Ingram, B.; Johnson, C. K.; Wilson, G. S. Anal. Chem. 2005, 77, 1963-1970.

(21) Li, W.; Wang, K.; Tan, W.; Ma, C.; Yang, X. Analyst 2007, 132, 107-113.

(22) Zhang, D.; Lu, M.; Wang, H. J. Am. Chem. Soc. 2011, 133, 9188-9191.

(23) Zou, M.; Chen, Y.; Xu, X.; Huang, H.; Liu, F.; Li, N. Biosens. Bioelectron. 2012, 32, 148-154.

(24) Wang, Y.; Killian, J.; Hamasaki, K.; Rando, R. R. Biochemistry 1996, 35, 12338-12346.

(25) Ruta, J.; Perrier, S.; Ravelet, C.; Fize, J.; Peyrin, E. Anal. Chem. 2009, 81, 7468-7473.

(26) Perrier, S.; Ravelet, C.; Guieu, V.; Roy, B.; Perigaud, C.; Peyrin, E. Biosens. Bioelectron. 2010, 25, 1652-1657. 
(27) Kidd, A.; Guieu, V.; Perrier, S.; Ravelet, C.; Peyrin, E. Anal. Bioanal. Chem. 2011, 401, 3229-3234.

(28) Cruz-Aguado, J. A.; Penner, G. Anal. Chem. 2008, 80, 88538855

(29) Zhu, Z.; Schmidt, T.; Mahrous, M.; Guieu, V.; Perrier, S.; Ravelet, C.; Peyrin, E. Anal. Chim. Acta 2011, 707, 191-196.

(30) Hafner, M.; Vianini, E.; Albertoni, B.; Marchetti, L.; Grune, I.; Gloeckner, C.; Famulok, M. Nat. Protoc. 2008, 3, 579-586.

(31) Cui, L.; Zou, Y.; Lin, N. H.; Zhu, Z.; Jenkins, G.; Yang, C. Y. Anal. Chem. 2012, 84, 5535-5541.

(32) Zhu, Z.; Ravelet, C.; Perrier, S.; Guieu, V.; Fiore, E.; Peyrin, E. Anal. Chem. 2012, 84, 7203-7211.

(33) Huang, Y.; Zhao, S. L.; Chen, Z. F.; Shi, M.; Liang, H. Chem. Commun. 2012, 48, 11877-11879.

(34) Liu, J.; Wang, C.; Jiang, Y.; Hu, Y.; Li, J.; Yang, S.; Li, Y.; Yang, R.; Tan, W.; Huang, C. Z. Anal. Chem. 2013, 85, 1424-1430.

(35) Yabuki, K.; Ouchi, T.; Ohya, Y. Supramol. Chem. 2003, 15, 149154.

(36) Heinlein, T.; Knemeyer, J.-P.; Piestert, O.; Sauer, M. J. Phys. Chem. B 2003, 107, 7957-7964.

(37) Torimura, M.; Kurata, S.; Yamada, K.; Yokomaku, T.; Kamagata, Y.; Kanagawa, T.; Kurane, R. Anal. Sci. 2001, 17, 155-160.

(38) Unruh, J. R.; Gokulrangan, G.; Wilson, G. S.; Johnson, C. K. Photochem. Photobiol. 2005, 81, 682-690.

(39) Unruh, J. R.; Gokulrangan, G.; Lushington, G. H.; Johnson, C. K.; Wilson, G. S. Biophys. J. 2005, 88, 3455-3465.

(40) Nazarenko, I.; Pires, R.; Lowe, B.; Obaidy, M.; Rashtchian, A. Nucleic Acids Res. 2002, 30, 2089-2195.

(41) Edman, L.; Mets, U.; Rigler, R. Proc. Natl. Acad. Sci. U.S.A. 1996, 93, 6710-6715.

(42) Zhang, D.; Zhao, Q.; Zhao, B.; Wang, H. Anal. Chem. 2012, 84, 3070-3074.

(43) Zhang, D.; Shen, H.; Li, G.; Zhao, B.; Yu, A.; Zhao, Q.; Wang, H. Anal. Chem. 2012, 84, 8088-8094.

(44) Monaci, L.; Palmisano, F. Anal. Bioanal. Chem. 2004, 378, 96103.

(45) Hayat, A.; Paniel, N.; Rhouati, A.; Marty, J.-L.; Barthelmebs, L. Food Control 2012, 26, 401-415.

(46) Cruz-Aguado, J. A.; Penner, G. J. Agric. Food Chem. 2008, 56, 10456-10461.

(47) Geng, X.; Zhang, D.; Wang, H.; Zhao, Q. Anal. Bioanal. Chem. 2013, 405, 2443-2449.

(48) Zhao, Q.; Geng, X.; Wang, H. Anal. Bioanal. Chem. 2013, 405, 6281-6286.

(49) Maragos, C. M. Mycotoxin Res. 2006, 22, 96-99.

(50) Kuang, H.; Chen, W.; Xu, D.; Xu, L.; Zhu, Y.; Liu, L.; Chu, H.; Peng, C.; Xu, C.; Zhu, S. Biosens. Bioelectron. 2010, 76, 710-716.

(51) Wang, L.; Ma, W.; Chen, W.; Liu, L.; Ma, W.; Zhu, Y.; Xu, L.; Kuang, H.; Xu, C. Biosens. Bioelectron. 2011, 26, 3059-3062.

(52) Bone, L.; Vidal, J. C.; Duato, P.; Castillo, J. R. Biosens. Bioelectron. 2011, 26, 3254-3259.

(53) Wang, L.; Chen, W.; Ma, W.; Liu, L.; Ma, W.; Zhao, Y.; Zhu, Y.; $\mathrm{Xu}$, L.; Kuang, H.; Xu, C. Chem. Commun. 2011, 47, 1574-1576.

(54) Chapuis-Hugon, F.; du Boisbaudry, A.; Madru, B.; Pichon, V. Anal. Bioanal. Chem. 2011, 400, 1199-1207.

(55) Yang, X. H.; Kong, W. J.; Yang, M. H.; Zhao, M.; Ouyang, Z. Chin. J. Anal. Chem. 2013, 41, 297-306.

(56) Girolamo, A. D.; McKeague, M.; Miller, J. D.; DeRosa, M. C.; Visconti, A. Food Chem. 2011, 127, 1378-1384.

(57) Zezza, F.; Longobardi, F.; Pascale, M.; Eremin, S. A.; Visconti, A. Anal. Bioanal. Chem. 2009, 395, 1317-1323.

(58) Shim, W. B.; Kolosova, A. Y.; Kim, Y. J.; Yang, Z. Y.; Park, S. J.; Eremin, S. A.; Lee, I. S.; Chung, D. H. Int. J. Food Sci. Technol. 2004, 39, 829-837. 